\title{
Floating drops and functions of bounded variation
}

\author{
Alan Elcrat* $\quad$ Ray Treinen ${ }^{\dagger}$
}

September 30, 2007

\begin{abstract}
A variational problem for three fluids in which gravitational and surface tension forces are in equilibrium is studied using sets of finite perimeter and functions of bounded variation. Existence theorems are proven which imply the existence of an axisymmetric floating drop. This problem has been studied previously as a free boundary problem for axisymmetric capillary surfaces by Elcrat, Neel and Siegel, but the general existence problem for arbitrary drop volumes was left open. The results presented here settle a version of this question.
\end{abstract}

\section{Introduction}

We consider here an equilibrium problem for capillary surfaces in which there are three fluids and, in general, three bounding surfaces. In configurations with rotationally symmetric containers, symmetry may be expected, and possible solutions to the energy minimization problem can be given by curves in the meridian plane. Minimization of the potential energy for this equilibrium problem then leads formally to a free boundary problem for axisymmetric capillary surfaces in which a sessile drop, an interior capillary surface, and an exterior capillary surface meet at a point. This differential equation problem has been studied extensively both theoretically and numerically by each of us [2], [3], [11]. A typical configuration is shown in Figure 1.

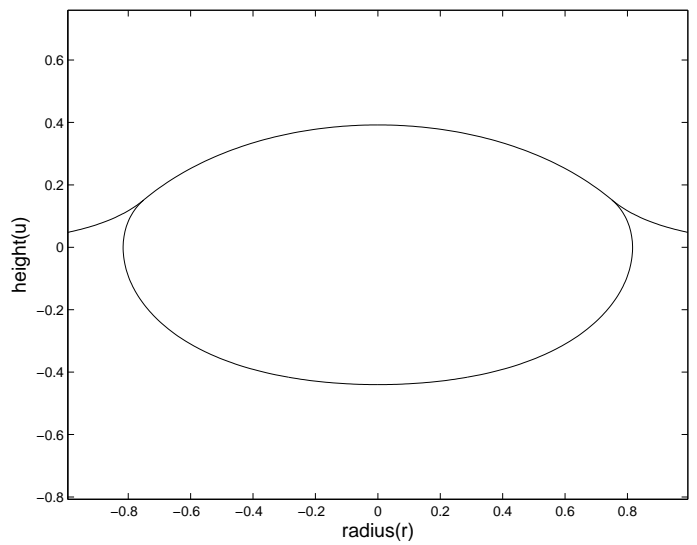

Figure 1: A typical floating drop.

\footnotetext{
${ }^{1}$ Department of Mathematics and Statistics, Wichita State University, Wichita, KS, 67218

${ }^{2}$ Department of Mathematics, Kansas State University, Manhattan, KS, 66502
} 
There are difficulties, however, in establishing existence in full generality using these methods. We reconsider the basic problem here using variational methods in spaces of functions of bounded variation. See for example, Ambrosio, Fusco, and Pallara [1], Evans and Gariepy [5], Giusti [7], Massari and Miranda [10], or Ziemer [14] for background on functions of bounded variation. See also Emmer [4], González [8] and Vogel [12], [13] for examples of just some of the many applications to capillary surfaces.

The general setup follows. Let $\Omega \subseteq \mathbb{R}^{3}$ be connected and open. The fluids are represented by the sets $E_{0}, E_{1}, E_{2}$, where $\Omega=\cup E_{i}$ and $E_{i} \cap E_{j}=\emptyset$ for $i \neq j$. Let $\rho_{0}, \rho_{1}, \rho_{2}$ be densities and such that $\rho_{0} \leq \rho_{1} \leq \rho_{2}$. Let $\sigma_{01}, \sigma_{02}, \sigma_{12}$ be surface tensions. Also, all the multiple indices are symmetric: eg. $\sigma_{i j}=\sigma_{j i}$. In order that the fluids remain separate we assume $\sigma_{01}, \sigma_{02}, \sigma_{12}>0$. The surface tensions will determine the contact angles with the wall and at any point where all three fluids meet. In order to be able to achieve force balance at any point of contact of three fluids we assume for $i, j=0,1,2$ each $\sigma_{i j}$ is less than the sum of the other two [2]. Here $S_{i j}:=\left\|\partial E_{i} \cap \partial E_{j} \cap \Omega\right\|_{H_{2}}$ are the surface areas of each surface and $g$ is the acceleration due to gravity.

Now

$$
\int_{\Omega}\left|D \chi_{E_{i}}\right|=\sum_{\substack{j=0 \\ j \neq i}}^{3} S_{i j}
$$

Define

$$
\begin{aligned}
\alpha_{0} & =\frac{1}{2}\left(\sigma_{01}+\sigma_{02}-\sigma_{12}\right) \\
\alpha_{1} & =\frac{1}{2}\left(\sigma_{01}+\sigma_{12}-\sigma_{02}\right) \\
\alpha_{2} & =\frac{1}{2}\left(\sigma_{02}+\sigma_{12}-\sigma_{01}\right)
\end{aligned}
$$

that is,

$$
\begin{aligned}
& \sigma_{01}=\alpha_{0}+\alpha_{1} \\
& \sigma_{12}=\alpha_{1}+\alpha_{2} \\
& \sigma_{02}=\alpha_{0}+\alpha_{2} .
\end{aligned}
$$

Formally define the energy functional to be

$$
\mathcal{F}_{\Omega}\left(E_{0}, E_{1}, E_{2}\right)=\sum_{i=0}^{2}\left(\alpha_{i} \int_{\Omega}\left|D \chi_{E_{i}}\right|+\rho_{i} g \int_{E_{i}} z d V\right) .
$$

We will sometimes refer to this as $\mathcal{F}$ when clear, or as $\mathcal{F}\left(E_{0}, E_{1}, E_{2} ; \Omega\right)$ when the subscript notation becomes cumbersome. Note that wetting energy terms could be included on $\partial \Omega$, however this generality is not needed in the solution of our problem.

Given a Borel set $E$, Giusti [7, Proposition 3.1] states that we may alter $E$ on a set of measure zero so that

$$
0<|E \cap B(x, \rho)|<\omega_{3} \rho^{3}
$$

for all $x \in \partial E$ and all $\rho>0$ where $\omega_{3}$ is the measure of the unit ball in $\mathbb{R}^{3}$. We will assume that all the sets in this paper are normalized in this way.

\section{The floating drop}

If (2) is computed for the configurations in [2], the value will not be finite because $E_{0}$ and $E_{2}$ have infinite volume. We will approach this problem by considering minimizers over a certain class of admissible sets 
in bounded subsets of $\mathbb{R}^{3}$ and using a limiting procedure we will show that there is a minimizer over a certain class of admissible sets to (2) restricted to arbitrary compact sets. Prescribe the volume $v_{1}$ of the "drop", that is the volume of the set $E_{1}$.

Definition 2.1 The triple $\left(E_{0}, E_{1}, E_{2}\right)$ is radially symmetric if $E_{1}=\{r<r(z)\}, E_{0}=\left\{r \in \cup I_{0}(z)\right\}$, and $E_{2}=\left\{r \in \cup I_{2}(z)\right\}$ for some intervals $I_{0}(z)$ and $I_{2}(z)$.

Theorem 2.2 If $\left(E_{0}, E_{1}, E_{2}\right)$ is the limit of radially symmetric triples, then $\left(E_{0}, E_{1}, E_{2}\right)$ is radially symmetric.

Proof. First consider $E_{1}$. Denote by $E_{1}^{n}$ a convergent sequence converging to $E_{1}$, where $E_{1}^{n}$ is part of a radially symmetric triple. Assume that $r_{n}(z)>0$ does not converge to $r(z)>0$ for $z$ in a set of positive measure. That is $\left|r_{n}-r_{m}\right| \geq \delta>0$ for a set of positive $z$-measure where $r_{n}, r_{m}>0$, for simplicity say $r_{n}-r_{m} \geq \delta>0$ and

$$
\left|E_{1}^{n}-E_{1}^{m}\right|=\int_{E_{1}^{n}-E_{1}^{m}} d x=\pi \int\left(\int_{r_{m}}^{r_{n}} r^{2} d r\right) d z \geq \frac{\pi}{3} \delta C>0
$$

for some positive constant $C$. This contradicts $E_{1}^{n} \rightarrow E_{1}$ in $L^{1}$, so $r_{n}(z) \rightarrow r(z)$ a.e. in $z$.

Then for $E_{0}$ consider $\left(a_{n}(z), b_{n}(z)\right)$ where $0<a_{n}, b_{n}<\infty$. If $E_{0}^{n}$ (part of a radially symmetric triple) is converging in $L^{1}$ and $a^{n}(z), b^{n}(z)$ are not converging, then we may assume $\left|a_{n}(z)-a_{m}(z)\right| \geq \delta>0$ and $\left|b_{n}(z)-b_{m}(z)\right| \geq \delta>0$ in a set of positive measure (passing to a subsequence if necessary). Then

$$
\begin{aligned}
\left|E_{0}^{n}-E_{0}^{m}\right| & =\int_{E_{0}^{n}-E_{0}^{m}} d V \\
& =\pi \int\left(\int_{a_{n}}^{b_{n}} r^{2} d r-\int_{a_{m}}^{b_{m}} r^{2} d r\right) d z \\
& =\frac{\pi}{3} \int\left(b_{n}^{3}-a_{n}^{3}-b_{m}^{3}+a_{m}^{3}\right) d z \geq \frac{\pi}{3} \delta C
\end{aligned}
$$

for some positive constant $C$. This contradicts $E_{0}^{n} \rightarrow E_{0}$ in $L^{1}$, so $a_{n}(z) \rightarrow a(z)$ and $b_{n}(z) \rightarrow b(z)$ a.e. in $z$. The case where the interval is semi-infinite is completely similar to the case for $E_{1}$.

The proof that $E_{2}$ is radially symmetric is the same as for $E_{0}$.

Definition 2.3 $A$ minimizer over radially symmetric, compact perturbations is the radially symmetric triple $\left(E_{0}, E_{1}, E_{2}\right)$, where each $E_{i}$ is a set of locally finite perimeter, if for a given compact set $K$ the following conditions hold:

1. $\int_{\Omega} \chi_{E_{1}}=v_{1}$,

2. For any radially symmetric $\left(A_{0}, A_{1}, A_{2}\right)$, where each $A_{i}$ is a set of locally finite perimeter and $\int_{\Omega} \chi_{A_{1}}=v_{1}$, such that $E_{i} \triangle A_{i} \subset K$, then $\left(E_{0}, E_{1}, E_{2}\right)$ satisfies $\mathcal{F}_{K}\left(E_{0}, E_{1}, E_{2}\right) \leq \mathcal{F}_{K}\left(A_{0}, A_{1}, A_{2}\right)$.

Here $\triangle$ is the symmetric difference: $E \triangle A=(E-A) \cup(A-E)$. We also require that the solutions are non-trivial, in that they have three fluids, and $E_{0}, E_{2}$ are both semi-infinite.

We will need the following special case of a result proved by Massari [9]:

Theorem 2.4 Let $\Omega$ be bounded, $\alpha_{i} \geq 0$ and $\sigma_{i j}>0$. Then there exists $\left(E_{0}, E_{1}, E_{2}\right)$, where $E_{i}$ are sets of finite perimeter, that minimizes $\mathcal{F}$ over

$$
\begin{aligned}
\mathcal{K}:= & \left\{\left(E_{0}, E_{1}, E_{2}\right) \mid \cup E_{i}=\Omega, E_{i} \cap E_{j}=\emptyset \text { for } i \neq j\right. \\
& \left|E_{i}\right|=v_{i}, \text { prescribed volumes, where } \sum v_{i}=|\Omega|, \\
& \text { and } \left.E_{i} \text { are sets of finite perimeter }\right\}
\end{aligned}
$$


The following corollary follows immediately from Massari's proof and Theorem 2.2.

Corollary 2.5 Let the hypotheses of Theorem 2.4 hold, and additionally assume that $\Omega$ is symmetric about the vertical axis. There exists radially symmetric $\left(E_{0}, E_{1}, E_{2}\right)$, where $E_{i}$ are sets of finite perimeter, that minimizes $\mathcal{F}$ over $\mathcal{K}^{\prime}:=\mathcal{K} \cap\left\{\left(E_{0}, E_{1}, E_{2}\right)\right.$ is radially symmetric $\}$.

We will need the following:

Theorem 2.6 The minimum for the functional $\mathcal{F}$ with all $\sigma_{i j}=0$ in a bounded, connected domain $\Omega$ over $\mathcal{K}$ is achieved by a configuration consisting of flat interfaces between the fluids, which are ordered decreasing with higher density.

Proof. Define the height of the interface between $E_{0}$ and $E_{1}$ to be $z_{1}=\sup \left\{z:(x, y, z) \in E_{1}\right\}$ and define the height of the interface between $E_{1}$ and $E_{2}$ to be $z_{2}=\sup \left\{z:(x, y, z) \in E_{2}\right\}$. Assume that there exists $\left(A_{0}, A_{1}, A_{2}\right)$ such that

$$
\mathcal{F}\left(A_{0}, A_{1}, A_{2}\right)<\mathcal{F}\left(E_{0}, E_{1}, E_{2}\right)-\delta
$$

for some $\delta>0$. This means that $\left(A_{0}, A_{1}, A_{2}\right)$ must differ from $\left(E_{0}, E_{1}, E_{2}\right)$ on a set of positive measure. Recall that we are asuming that the sets are normalized as in (3). Thus not all of the following are true:

1. $A_{0}=E_{0}$,

2. $A_{1}=E_{1}$,

3. $A_{2}=E_{2}$,

where we are identifying sets equal up to measure zero.

First, assume that 1 . is not true. Then

$$
\tilde{A}_{0}=\left\{z<z_{1}\right\} \cap A_{0}
$$

has positive measure and must displace some fluid below $z=z_{1}$. Then the volume constraints force one or both of 2. or 3. to be false. Thus some of $A_{1}$ or $A_{2}$ or both must be above $z=z_{1}$. Label

$$
\begin{aligned}
& \tilde{A}_{1}=\left\{z>z_{1}\right\} \cap A_{1} \\
& \tilde{A}_{2}=\left\{z>z_{1}\right\} \cap A_{2} .
\end{aligned}
$$

One of $\tilde{A}_{1}$ or $\tilde{A}_{2}$ must have positive measure. By volume considerations $\left|\tilde{A}_{1}\right| \leq\left|\tilde{A}_{0}\right|$ and $\left|\tilde{A}_{2}\right|=\left|\tilde{A}_{0}\right|-\left|\tilde{A}_{1}\right|$. Select $A \subset \tilde{A}_{0}$ such that $|A|=\left|\tilde{A}_{1}\right|$ and $B \subset \tilde{A}_{0}-A$ such that $|B|=\left|\tilde{A}_{2}\right|$. Then switch: identify $A$ with $A_{1}$ and $\tilde{A}_{1}$ with $A_{0}$; identify $B$ with $A_{2}$ and $\tilde{A}_{2}$ with $A_{0}$. This lowers the value of the energy functional.

Note that 1 . is now true. Volume considerations now imply that 2 . is false if and only if 3 . is false. Now label

$$
\begin{aligned}
& \tilde{A}_{1}=\left\{z<z_{2}\right\} \cap A_{1} \\
& \tilde{A}_{2}=\left\{z>z_{2}\right\} \cap A_{2} .
\end{aligned}
$$

We deduce that $\left|\tilde{A}_{1}\right|=\left|\tilde{A}_{2}\right|$ and we switch by identifying $\tilde{A}_{1}$ with $A_{2}$ and $\tilde{A}_{2}$ with $A_{1}$. This lowers the value of the energy functional and supplies a contradiction as our new sets $A_{0}, A_{1}, A_{2}$ are equal to $E_{0}, E_{1}, E_{2}$ respectively. The need for $\Omega$ to be connected is so that the fluids can move freely under perturbations.

The main result of the paper is 
Theorem 2.7 For a given volume $v_{1}$ there exists radially symmetric $\left(E_{0}, E_{1}, E_{2}\right)$ that minimizes $\mathcal{F}_{K}$ over compact symmetric perturbations in $\Omega=\mathbb{R}^{2} \times(-T, T)$ for any compact set $K$.

Proof. Consider the cylinder $C(n)$, with $n$ large enough that $v_{1}<|C(n)|$. Set $v_{0}^{n}=\frac{1}{2}\left(|C(n)|-v_{1}\right)$ and $v_{2}^{n}=\frac{1}{2}\left(|C(n)|-v_{1}\right)$ and consider the minimizer of the functional $\mathcal{F}$ over $\mathcal{K}^{\prime}$ in the bounded domain $C(n)$. We denote this minimizer in this cylinder as $\left(E_{0}^{n}, E_{1}^{n}, E_{2}^{n}\right)$ and note the triple is radial, as follows from Corollary 2.5.

Our first goal is to find a convergent subsequence of $E_{1}^{n}$ as $n \rightarrow \infty$. We will need to following two lemmas to show this.

Lemma $2.8 E_{1}^{n}$ is uniformly bounded in $\Omega$ for all $n$.

Proof. To show that $E_{1}^{n}$ is uniformly bounded in $\{-T<z<T\}$ we first assume otherwise. That is for every $a>0$ there is an $n$ large enough that $r_{n}>a$ where $r_{n}$ is the maximal radius of $E_{1}^{n}$.

Now

$$
\begin{aligned}
& \sum_{i=0}^{2}\left(\alpha_{i} \int_{C(n)}\left|D \chi_{E_{i}^{n}}\right|+\rho_{i} g \int_{C(n)} \chi_{E_{i}^{n}} z d V\right) \\
> & \alpha_{1} \pi r_{n}^{2}+\pi \sigma_{02} n^{2}+\sum_{i=0}^{2} \rho_{i} g \int_{C(n)} \chi_{E_{i}^{n}} z d V \\
> & \alpha_{1} \pi r_{n}^{2}+\pi \sigma_{02} n^{2}+\frac{1}{2} g \pi^{2} n^{4}\left\{\frac{\rho_{0}}{v_{0}^{2}}\left(2 v_{0} T-\pi n^{2}\right)+\frac{\rho_{2}}{v_{2}^{2}}\left(\pi n^{2}-2 v_{2} T\right)+\right. \\
& \left.\frac{\rho_{1}}{v_{0}^{2} v_{2}^{2}}\left[2 T\left(v_{0}-v_{2}\right) v_{0} v_{2}+\pi n^{2}\left(v_{2}^{2}-v_{0}^{2}\right)\right]\right\} \\
= & \alpha_{1} \pi r_{n}^{2}+\pi \sigma_{02} n^{2}+\frac{g \pi^{2} n^{4}}{2 v_{2}}\left(\rho_{2}-\rho_{0}\right)\left(\frac{\pi n^{2}}{v_{2}}-2 T\right)
\end{aligned}
$$

as we replace the surface area terms of the drop with the area of a disc and the the remaining surface area terms with the area of a washer. Clearly this reduces the surface area. The gravity terms are reduced by replacing them with value of $\mathcal{F}$ from the sets from Theorem 2.6 with $v_{0}=v_{2}$. This is no longer an admissible configuration, but it is a lower bound.

Next we see that

$$
\begin{aligned}
& \sum_{i=0}^{2}\left(\alpha_{i} \int_{C(n)}\left|D \chi_{E_{i}^{n}}\right|+\rho_{i} g \int_{C(n)} \chi_{E_{i}^{n}} z d V\right) \\
\leq & \pi r_{1}^{2}\left(\sigma_{01}+\sigma_{12}\right)+2 \pi r_{1}\left(\frac{1}{2} \frac{v_{1}}{\pi r_{1}^{2}}\right)\left(\sigma_{01}+\sigma_{12}\right)+\pi \sigma_{02}\left(n^{2}-r_{1}^{2}\right)+\sum_{i=0}^{2} \rho_{i} g \int_{C(n)} \chi_{A_{i}^{n}} z d V \\
= & \alpha_{1} \pi r_{1}^{2}+\pi \sigma_{02} n^{2}+\frac{v_{1}}{r_{1}}\left(\sigma_{01}+\sigma_{12}\right)+\left(\rho_{2}-\rho_{0}\right) g \frac{\pi}{8}\left(r_{1}^{2} h^{2}-4 T^{2} n^{2}\right)
\end{aligned}
$$

where $A_{1}=A_{1}^{n}$ is the cylinder of radius $r_{1}$ and height following from $v_{1}=\pi r_{1}^{2} h$ that is divided evenly by the $x y$-plane, $A_{2}^{n}=\left(C(n)-A_{1}\right) \cap\{z<0\}$ and $A_{0}^{n}=C(n)-\left(A_{1} \cup A_{2}^{n}\right)$. The sets $A_{0}^{n}, A_{1}, A_{2}^{n}$ form an admissible configuration for $C(n)$ and so have energy no less than that of the minimizer. The gravity terms reduce here due to the symmetry of the configuration about the $x y$-plane and integration.

Combining these two inequalities we obtain

$$
\begin{aligned}
& \alpha_{1} \pi r_{n}^{2}+\pi \sigma_{02} n^{2}+\frac{g \pi^{2} n^{4}}{2 v_{2}}\left(\rho_{2}-\rho_{0}\right)\left(\frac{\pi n^{2}}{v_{2}}-2 T\right) \\
< & \alpha_{1} \pi r_{1}^{2}+\pi \sigma_{02} n^{2}+\frac{v_{1}}{r_{1}}\left(\sigma_{01}+\sigma_{12}\right)+\left(\rho_{2}-\rho_{0}\right) g \frac{\pi}{8}\left(r_{1}^{2} h^{2}-4 T^{2} n^{2}\right)
\end{aligned}
$$




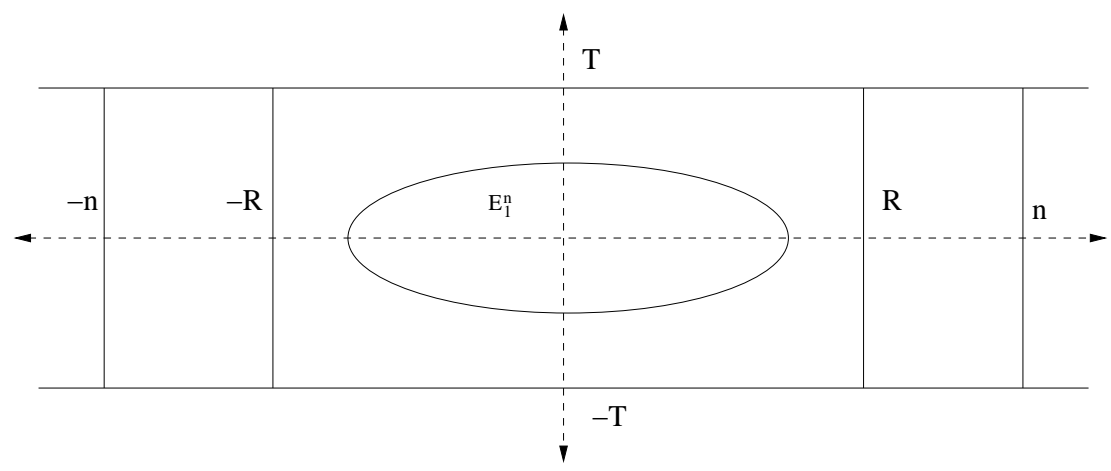

Figure 2: The set $E_{1}^{n}$ bounded in the cylinder of radius $R$, height $2 T$, independently of $C(n)$.

which, as

$$
v_{2}=\frac{\pi n^{2}}{2}\left(2 T-\frac{\pi n^{2}}{v_{1}}\right)
$$

we can rewrite as

$$
\begin{aligned}
& \alpha_{1} \pi\left(r_{n}^{2}-r_{1}^{2}\right)-\frac{v_{1}}{r_{1}}\left(\sigma_{01}+\sigma_{12}\right) \\
< & \left(\rho_{2}-\rho_{0}\right) g \frac{\pi}{8}\left(r_{1}^{2} h^{2}-4 T^{2} n^{2}\right)-\frac{g \pi^{2} n^{4}}{2 v_{2}}\left(\rho_{2}-\rho_{0}\right)\left(\frac{\pi n^{2}}{v_{2}}-2 T\right) \\
= & \left(\rho_{2}-\rho_{0}\right) g\left[\frac{\pi}{8} r_{1}^{2} h^{2}-\frac{\pi}{2} n^{2} T^{2}-\frac{\pi n^{2}}{2 T-\frac{\pi n^{2}}{v_{1}}}\left(\frac{2}{2 T-\frac{\pi n^{2}}{v_{1}}}-2 T\right)\right]
\end{aligned}
$$

which goes to $-\infty$ as $n \rightarrow \infty$. This contradicts the assumption that $r_{n} \rightarrow \infty$. Thus we know that there is a large enough radius $R$ where $E_{1}^{n} \subset C(R)$ for all $n$. See Figure 2.

Lemma $2.9 \sup _{n} \int_{C(R)}\left|D \chi_{E_{1}^{n}}\right|<\infty$.

Proof. Note that $\mathcal{F}_{C(n)}\left(E_{0}^{n}, E_{1}^{n}, E_{2}^{n}\right) \leq \mathcal{F}_{C(n)}\left(A_{0}^{n}, A_{1}^{n}, A_{2}^{n}\right)$ for all admissible $A$ 's. In particular we can set $A_{1}=A_{1}^{n}$ to be the cylinder of height $2 T$ with radius $r_{1}$ that satisfies $v_{1}=2 \pi r_{1}^{2} T$. Since $E_{1}^{n} \subset C(R)$ we may set $A_{2}^{n}=E_{2}^{n}$ and $A_{0}^{n}=E_{0}^{n}$ outside of $C(R)$ and within $C(R)-C\left(r_{1}\right)$ set $A_{2}^{n}$ to be everything below a height $h_{n}$ which is determined to get the volumes correct. 
From the inequality for the energy we obtain, primarily by bounding the trace on $\partial C(R)$ :

$$
\begin{aligned}
& \alpha_{1} \int_{C(R)}\left|D \chi_{E_{1}^{n}}\right| \\
\leq & \sum_{i=0}^{2}\left(\alpha_{i} \int_{C(n)}\left|D \chi_{A_{i}^{n}}\right|+\rho_{i} g \int_{C(n)} \chi_{A_{i}^{n}} z d V-\rho_{i} g \int_{C(n)} \chi_{E_{i}^{n}} z d V\right) \\
& -\alpha_{0} \int_{C(n)}\left|D \chi_{E_{0}^{n}}\right|-\alpha_{2} \int_{C(n)}\left|D \chi_{E_{2}^{n}}\right| \\
< & \alpha_{1} 4 \pi r_{1} T+\alpha_{0}\left(8 \pi T\left(r_{1}+R\right)+2 \pi\left(R^{2}-r_{1}^{2}\right)-\int_{C(R)}\left|D \chi_{E_{0}^{n}}\right|\right) \\
& +\alpha_{2}\left(8 \pi T\left(r_{1}+R\right)+2 \pi\left(R^{2}-r_{1}^{2}\right)-\int_{C(R)}\left|D \chi_{E_{2}^{n}}\right|\right)+\sum_{i=0}^{2} \rho_{i} g \int_{C(R)}\left(z \chi_{A_{i}^{n}}-z \chi_{E_{i}^{n}}\right) d V \\
< & 4 \pi \alpha_{1} r_{1} T+2 \pi\left(\alpha_{0}+\alpha_{2}\right)\left(R+r_{1}\right)\left(4 T+R-r_{1}\right)+2\left(\Sigma \rho_{i}\right) g \int_{C(R)} z d V .
\end{aligned}
$$

The right side does not depend on $n$, thus taking supremum with respect to $n$ from both sides implies $\sup _{n} \int_{C(R)}\left|D \chi_{E_{1}^{n}}\right|<\infty$.

Set $\Omega=C(R)$. As $E_{1}^{n} \subset \Omega$ which is bounded and open, the general compactness theorem for $B V$ functions applies and this implies the existence of a limit to a subsequence of $E_{1}^{n}$. Denote $E_{1}=\lim E_{1}^{n_{j}}$. Recall that $\left(E_{0}^{n}, E_{1}^{n}, E_{2}^{n}\right)$ is the minimizer on the cylinder $C(n)$. Next we show that $E_{0}^{n}$ and $E_{2}^{n}$ have convergent subsequences with limits $E_{0}, E_{2}$ such that $\cup E_{i}=\Omega$.

First extend each $E_{0}^{n}$ to include the set $\tilde{A}_{0}^{n}=\left\{(x, y, z): n<\sqrt{x^{2}+y^{2}}, 0<z<T\right\}$. Similarly extend $E_{2}^{n}$ to include $\tilde{A}_{2}^{n}=\left\{(x, y, z): n<\sqrt{x^{2}+y^{2}},-T<z<0\right\}$. Now each of $E_{0}^{n}$ and $E_{2}^{n}$ are defined outside of $C(n)$, and can be components of an admissible configuration on larger cylinders. Consider a cylinder $C(k)$ of radius $k$. Denote restrictions of $E_{0}^{n}$ to $C(k)$ by $E_{0}^{n}(k):=E_{0}^{n} \cap C(k)$ and similarly for $E_{2}^{n}$.

Lemma 2.10 The sequences of sets $E_{0}^{n}(k), E_{2}^{n}(k)$ have convergent subsequences on the cylinder $C(k)$, denoting the limiting sets by $E_{0}(k)$ and $E_{2}(k)$ respectively.

Proof. Now

$$
\begin{aligned}
\mathcal{F}\left(E_{0}^{n}, E_{1}^{n}, E_{2}^{n} ; C(n)\right) & =\mathcal{F}\left(E_{0}^{n}, E_{1}^{n}, E_{2}^{n} ; C(k)\right)+\mathcal{F}\left(E_{0}^{n}, E_{1}^{n}, E_{2}^{n} ; C(n)-\overline{C(k)}\right) \\
& +\sum_{i=0}^{2} \alpha_{i} \int_{\partial C(k) \cap \Omega}\left(T^{+} \chi_{E_{i}^{n}}-T^{-} \chi_{E_{i}^{n}}\right) d H_{2} .
\end{aligned}
$$

Observing (25) becomes an inequality $<$ if the sum is replaced with $2 \pi k T\left(\alpha_{0}+\alpha_{1}+\alpha_{2}\right)$, and if $k>R$ then we may use $2 \pi k T\left(\alpha_{0}+\alpha_{2}\right)$, then as $\left(E_{0}^{n}, E_{1}^{n}, E_{2}^{n}\right)$ is a minimizer, for sets $\left(E_{0}^{k}, E_{1}^{k}, E_{2}^{k}\right)$ that minimize $\mathcal{F}$ on $C(k)$, we have $\mathcal{F}\left(E_{0}^{n}, E_{1}^{n}, E_{2}^{n} ; C(n)\right) \leq \mathcal{F}\left(E_{0}^{k}, E_{1}^{k}, E_{2}^{k} ; C(n)\right)$. Then writing everything out explicitly: 


$$
\begin{aligned}
& \sum_{i=0}^{2} \alpha_{i} \int_{C(k)}\left|D \chi_{E_{i}^{n}}\right|+\sum_{i=0}^{2} \alpha_{i} \int_{C(n)-\overline{C(k)}}\left|D \chi_{E_{i}^{n}}\right|+\sum_{i=0}^{2} \alpha_{i} \int_{\partial C(k) \cap \Omega}\left(T^{+} \chi_{E_{i}^{n}}-T^{-} \chi_{E_{i}^{n}}\right) d H_{2} \\
+ & \sum_{i=0}^{2} \rho_{i} g \int_{C(k)} \chi_{E_{i}^{n}} z d V+\sum_{i=0}^{2} \rho_{i} g \int_{C(n)-\overline{C(k)}} \chi_{E_{i}^{n}} z d V \\
< & \sum_{i=0}^{2} \alpha_{i} \int_{C(k)}\left|D \chi_{E_{i}^{k}}\right|+\sum_{i=0}^{2} \alpha_{i} \int_{C(n)-\overline{C(k)}}\left|D \chi_{\tilde{A}_{i}^{k}}\right|+4 \pi k T\left(\alpha_{0}+\alpha_{2}\right) \\
+ & \sum_{i=0}^{2} \rho_{i} g \int_{C(k)} \chi_{E_{i}^{k}} z d V+\sum_{i=0}^{2} \rho_{i} g \int_{C(n)-\overline{C(k)}} \chi_{\tilde{A}_{i}^{k}} z d V .
\end{aligned}
$$

Using $\alpha_{1} \int_{C(k)}\left|D \chi_{E_{1}^{n}}\right|>0$, we rearrange (26):

$$
\begin{aligned}
\alpha_{0} \int_{C(k)}\left|D \chi_{E_{0}^{n}}\right|+\alpha_{2} \int_{C(k)}\left|D \chi_{E_{2}^{n}}\right| & <\sum_{i=0}^{2} \alpha_{i} \int_{C(k)}\left|D \chi_{E_{i}^{k}}\right|+4 \pi k T\left(\alpha_{0}+\alpha_{2}\right) \\
& +\sum_{i=0}^{2} \alpha_{i}\left(\int_{C(n)-\overline{C(k)}}\left|D \chi_{\tilde{A}_{i}^{k}}\right|-\int_{C(n)-\overline{C(k)}}\left|D \chi_{E_{i}^{n}}\right|\right) \\
& +\sum_{i=0}^{2} \rho_{i} g\left(\int_{C(k)} \chi_{E_{i}^{k}} z d V-\int_{C(k)} \chi_{E_{i}^{n}} z d V\right) \\
& +\sum_{i=0}^{2} \rho_{i} g\left(\int_{C(n)-\overline{C(k)}} \chi_{\tilde{A}_{i}^{k}} z d V-\int_{C(n)-\overline{C(k)}} \chi_{E_{i}^{n}} z d V\right) .
\end{aligned}
$$

The terms on the right side of line (27) are finite as $E_{i}^{k}$ are minimizers. The terms in (28) are negative because the first term is the smallest such configuration in $C(n)-\overline{C(k)}$. In (29) observe

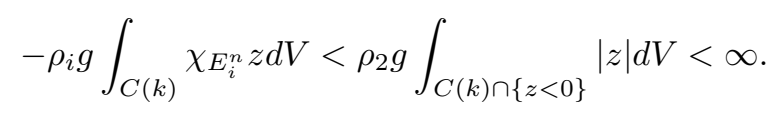

Finally, as $\tilde{A}_{i}^{k}$ are the minimizers to the "pure gravity" functional, Lemma 2.6, the terms in (30) are negative. Thus

$$
\begin{aligned}
\alpha_{0} \int_{C(k)}\left|D \chi_{E_{0}^{n}}\right|+\alpha_{2} \int_{C(k)}\left|D \chi_{E_{2}^{n}}\right| & <\sum_{i=0}^{2} \alpha_{i} \int_{C(k)}\left|D \chi_{E_{i}^{k}}\right|+\sum_{i=0}^{2} \rho_{i} g \int_{C(k)} \chi_{E_{i}^{k}} z d V \\
& +4 \pi k T\left(\alpha_{0}+\alpha_{2}\right)+3 \rho_{2} g \int_{C(k) \cap\{z<0\}}|z| d V
\end{aligned}
$$

and as the right side of this inequality does not depend on $n$ we have established a uniform bound for $\int_{C(k)}\left|D \chi_{E_{0}^{n}}\right|$ and $\int_{C(k)}\left|D \chi_{E_{2}^{n}}\right|$. Since $E_{i}^{n}(k) \subset C(k)$ we may apply the compactness theorem to claim the existence of a convergent subsequence for both $E_{0}^{n}(k)$ and $E_{2}^{n}(k)$. Denote the limits of these subsequences as $E_{0}(k)$ and $E_{2}(k)$ respectively.

Consider cylinders $C\left(k_{i}\right)$ and $C\left(k_{j}\right)$ where $k_{i}<k_{j}$, and consider $E_{0}^{n}\left(k_{i}\right), E_{0}^{n}\left(k_{j}\right) \cap C\left(k_{i}\right)$. It follows from the above argument that there exists a subsequence $E_{0}^{n_{m}}\left(k_{j}\right)$ that converges on $C\left(k_{i}\right)$. There is 
a convergent subsequence of that subsequence: $E_{0}^{n_{m_{l}}}\left(k_{j}\right)$ by the above argument, and it converges on $C\left(k_{j}\right)$. That is, while $E_{0}^{n_{m_{l}}}\left(k_{i}\right) \rightarrow E_{0}\left(k_{j}\right)$ we also have $E_{0}^{n_{m_{l}}}\left(k_{j}\right) \rightarrow E_{0}\left(k_{j}\right)$. That is, we may pick the limiting sets that coincide on the smaller of the two cylinders. Assume this is the case.

Lemma 2.11 The sequence of sets $E_{0}(k)$ and $E_{2}(k)$ both have convergent subsequences as $k \rightarrow \infty$. Denote these limits as $E_{0}$ and $E_{2}$ respectively. The sets $E_{0}, E_{2}$ are sets of locally finite perimeter.

Proof. Explicitly working out a diagonalization argument, a convergent subsequence for all cylinders $C(k)$ is found. Let $C\left(k_{1}\right), C\left(k_{2}\right), \ldots$ be a sequence of cylinders with radii $k_{i}$, natural numbers, increasing for $i=1,2, \ldots$. Consider first the subsequence $n_{m_{1}}, n_{m_{2}}, \ldots$ where

$$
E_{0}^{n_{m_{1}}}\left(k_{1}\right), E_{0}^{n_{m_{2}}}\left(k_{1}\right), \ldots \quad \text { converges on } C\left(k_{1}\right)
$$

then take $n_{p_{1}}, n_{p_{2}}, \ldots$ as a subsequence of $n_{m_{1}}, n_{m_{2}}, \ldots$ where

$$
E_{0}^{n_{p_{1}}}\left(k_{2}\right), E_{0}^{n_{p_{2}}}\left(k_{2}\right), \ldots \quad \text { converges on } C\left(k_{2}\right)
$$

Then take $n_{r_{1}}, n_{r_{2}}, \ldots$ as a subsequence of $n_{p_{1}}, n_{p_{2}}, \ldots$ where

$$
E_{0}^{n_{r_{1}}}\left(k_{3}\right), E_{0}^{n_{r_{2}}}\left(k_{3}\right), \ldots \quad \text { converges on } C\left(k_{3}\right)
$$

and so on. We see that for each row the convergent subsequence is a subsequence of the previous row's convergent subsequence. Thus the diagonal subsequence

$$
E_{0}^{n_{m_{1}}}(k), E_{0}^{n_{p_{2}}}(k), E_{0}^{n_{r_{3}}}(k), \ldots \quad \text { converges on } C(k)
$$

for every $k=k_{i}$. Since these limiting sets coincide for every cylinder $C(k)$, there exists $E_{0}=\lim _{k \rightarrow \infty} E_{0}(k)$. It is immediate that $E_{0}$ is a set of locally finite perimeter. A similar argument will produce a convergent subsequence for $E_{2}^{n}(k)$ and a set of locally finite perimeter $E_{2}=\lim _{k \rightarrow \infty} E_{2}(k)$.

Claim: This configuration $\left(E_{0}, E_{1}, E_{2}\right)$ is the minimizer we seek. Since we have $\int_{\Omega} \chi_{E_{1}}=v_{1}$ we have satisfied the first part of the definition. It remains verify that $\left(E_{0}, E_{1}, E_{2}\right)$ is a "local" minimizer.

Consider a compact set $K$ and assume that there exists an admissible configuration $\left(A_{0}, A_{1}, A_{2}\right)$ such that for some $\delta>0$

$$
\mathcal{F}_{K}\left(A_{0}, A_{1}, A_{2}\right)<\mathcal{F}_{K}\left(E_{0}, E_{1}, E_{2}\right)-\delta .
$$

Now outside of $K$ we have $A_{i}=E_{i}$. Also as $K$ is compact there is a cylinder $C(n)$ that contains $K$, where $n$ is from the convergent subsequence constructed above. So for minimizers $\left(E_{0}^{n}, E_{1}^{n}, E_{2}^{n}\right)$ on the cylinder $C(n, 2 T)$, and for some $k>n$ from the convergent subsequence noted above, we have

$$
\begin{aligned}
& \mathcal{F}_{K}\left(A_{0}, A_{1}, A_{2}\right)-\mathcal{F}_{K}\left(E_{0}, E_{1}, E_{2}\right) \\
= & \mathcal{F}_{C(n)}\left(A_{0}, A_{1}, A_{2}\right)-\mathcal{F}_{C(n)}\left(E_{0}, E_{1}, E_{2}\right) \\
= & \mathcal{F}_{C(n)}\left(A_{0}, A_{1}, A_{2}\right)-\mathcal{F}_{C(n)}\left(E_{0}^{n}, E_{1}^{n}, E_{2}^{n}\right)+\mathcal{F}_{C(n)}\left(E_{0}^{n}, E_{1}^{n}, E_{2}^{n}\right) \\
- & \mathcal{F}_{C(n)}\left(E_{0}^{k}, E_{1}^{k}, E_{2}^{k}\right)+\mathcal{F}_{C(n)}\left(E_{0}^{k}, E_{1}^{k}, E_{2}^{k}\right)-\mathcal{F}_{C(n)}\left(E_{0}, E_{1}, E_{2}\right) .
\end{aligned}
$$

Note that $\mathcal{F}_{C(n)}\left(A_{0}, A_{1}, A_{2}\right)-\mathcal{F}_{C(n)}\left(E_{0}^{n}, E_{1}^{n}, E_{2}^{n}\right) \geq 0$ as $\left(E_{0}^{n}, E_{1}^{n}, E_{2}^{n}\right)$ is the energy minimizer on $C(n, 2 T)$. For $k, n$ large enough it follows that

$$
\left|\mathcal{F}_{C(n)}\left(E_{0}^{n}, E_{1}^{n}, E_{2}^{n}\right)-\mathcal{F}_{C(n)}\left(E_{0}^{k}, E_{1}^{k}, E_{2}^{k}\right)\right|<\delta / 2,
$$

as $B V(C(n))$ is a Banach space under the $B V$ norm, and $C(n)$ is bounded (for that fixed, large $n$ ). Lastly, note that for $k$ large enough

$$
\left|\mathcal{F}_{C(n)}\left(E_{0}^{k}, E_{1}^{k}, E_{2}^{k}\right)-\mathcal{F}_{C(n)}\left(E_{0}, E_{1}, E_{2}\right)\right|<\delta / 2 .
$$


Putting this together, we have contradicted (37). Note that Corollary 2.5 applies in this case, thus $\left(E_{0}, E_{1}, E_{2}\right)$ is radial. This completes the proof of the theorem.

This establishes the existence of an axisymmetric minimizer for the variational problem studied in [2]. To make the connection with that work complete regularity of this solution should be established. It should be noted that it follows from the regularity theory for the capillary problem that the interfaces will be analytic in any ball that contains only two fluids. In addition the finite domain is undoubtedly connected when $\mathrm{T}$ is large. These matters are left for a subsequent work.

The authors would like to thank Luigi Ambrosio and Henry Wente for helpful comments. The second author completed portions of this work while at both the University of Toledo and Wichita State University.

\section{References}

[1] Luigi Ambrosio, Nicola Fusco, and Diego Pallara, Functions of bounded variation and free discontinuity problems, Oxford Mathematical Monographs, The Clarendon Press Oxford University Press, New York, 2000.MR1857292 (2003a:49002)

[2] Alan Elcrat, Robert Neel, and David Siegel, Equilibrium configurations for a floating drop, J. Math. Fluid Mech. 6 (2004), no. 4, 405-429.MR2101889 (2005j:76017)

[3] Alan Elcrat and Ray Treinen, Numerical results for a floating drop, Discrete and Continuous Dynamical Systems Supplements (2005), 241-249.

[4] Michele Emmer, Esistenza, unicità e regolarità nelle superfici de equilibrio nei capillari, Ann. Univ. Ferrara Sez. VII (N.S.) 18 (1973), 79-94 (Italian, with English summary). MR 0336507 (49 \#1281)

[5] Lawrence C. Evans and Ronald F. Gariepy, Measure theory and fine properties of functions, Studies in Advanced Mathematics, CRC Press, Boca Raton, FL, 1992.MR1158660 (93f:28001)

[6] Robert Finn, Equilibrium capillary surfaces, Grundlehren der Mathematischen Wissenschaften [Fundamental Principles of Mathematical Sciences], vol. 284, Springer-Verlag, New York, 1986.MR816345 (88f:49001)

[7] Enrico Giusti, Minimal surfaces and functions of bounded variation, Monographs in Mathematics, vol. 80, Birkhäuser Verlag, Basel, 1984.MR775682 (87a:58041)

[8] Eduardo H. A. Gonzalez, Sul problema della goccia appoggiata, Rend. Sem. Mat. Univ. Padova 55 (1976), $289-302$ (Italian).MR0493670 (58 \#12648)

[9] U. Massari, The parametric problem of capillarity: the case of two and three fluids, Astérisque (1984), no. 118, 197-203 (English, with French summary).MR761749 (86b:49061)

[10] Umberto Massari and Mario Miranda, Minimal surfaces of codimension one, North-Holland Mathematics Studies, vol. 91, North-Holland Publishing Co., Amsterdam, 1984.MR795963 (87f:49058)

[11] Ray Treinen, A study of floating drops, Wichita State Univ., 2004.

[12] Thomas I. Vogel, Unbounded parametric surfaces of prescribed mean curvature, Indiana Univ. Math. J. 31 (1982), no. 2, 281-288.MR648178 (83i:53016)

[13] Uniqueness for certain surfaces of prescribed mean curvature, Pacific J. Math. 134 (1988), no. 1, 197207.MR953508 (89i:53011)

[14] William P. Ziemer, Weakly differentiable functions, Graduate Texts in Mathematics, vol. 120, Springer-Verlag, New York, 1989.MR1014685 (91e:46046) 